msh-mss Mathématiques et sciences humaines

178 | Été 2007

Art, mathématiques, langage et émotion

\title{
Les unités sémiotiques temporelles
}

Temporal Semiotic Units

Jean Favory

\section{OpenEdition}

Journals

Édition électronique

URL : http://journals.openedition.org/msh/4192

DOI : $10.4000 /$ msh.4192

ISSN : 1950-6821

\section{Éditeur}

Centre d'analyse et de mathématique sociales de l'EHESS

\section{Édition imprimée}

Date de publication : 1 juillet 2007

Pagination : 51-55

ISSN : 0987-6936

\section{Référence électronique}

Jean Favory, "Les unités sémiotiques temporelles », Mathématiques et sciences humaines [En ligne],

178 | Été 2007, mis en ligne le 21 septembre 2007, consulté le 23 juillet 2020. URL : http://

journals.openedition.org/msh/4192 ; DOI : https://doi.org/10.4000/msh.4192 


\title{
LES UNITÉS SÉMIOTIQUES TEMPORELLES ${ }^{1}$
}

\author{
Jean FAVORY ${ }^{2}$
}

\begin{abstract}
RÉSUMÉ - Les Unités Sémiotiques Temporelles sont des fragments sonores qui, même hors de leur contexte musical, possèdent une signification temporelle due à leur organisation. Temporelles

MOTS-CLÉS - Analyse musicale, Morphologie sonore, Objets musicaux, Unités Sémiotiques

SUMMARY - Temporal Semiotic Units

Temporal Semiotic Units are sound fragments that, even out of their musical context, have a temporal signification due to their morphological organization.
\end{abstract}

KEY-WORDS - Musical analysis, Musical Objects, Sound morphology, Temporal Semiotic Units

Il est entendu de considérer que la musique des compositeurs d'aujourd'hui ne fait plus référence à une base commune à l'expression de la création musicale, comme l'était le système tonal jusqu'au tournant du $\mathrm{XIX}^{\mathrm{e}}$ siècle. Chaque compositeur, ou presque, a son système propre. En tout cas pour les musiques qui ne sont pas celles de la consommation de masse. Pour ne pas parler de musiques savantes. Cela a conduit à une certaine forme de rejet des auditeurs face à la création musicale pour des raisons de rupture de langage, donc, mais aussi pour des raisons économiques aussi bien que pédagogiques. ici.

Ce sont les raisons pédagogiques qui ont motivé le travail de recherche présenté

Les membres du Laboratoire Musique et Informatique de Marseille ${ }^{3}$, qui en ont eu l'initiative, sont pour la plupart des pédagogues, en plus d'être des créateurs. Et comme tels, ils se posent la question sur la façon d'aborder le répertoire musical contemporain avec leurs élèves. Principalement, à l'origine, celui des musiques électroacoustiques, ces musiques sur supports n'utilisant pas d'instruments mais un matériau sonore enregistré, synthétisé, transformé par des traitements, mis en forme par des opérations de montage et de mixage, puis diffusés sur un orchestre de haut-parleurs. Comment, face à l'absence de théorisation dans ces musiques sur ce qu'il convient de faire, comment, lors de l'écoute critique des travaux des élèves apprentis compositeurs, leur faire comprendre

\footnotetext{
${ }^{1}$ D'après un travail de François Delalande, Jean Favory, Marcel Formosa, Marcel Frémiot, Pascal Gobin, Jacques Mandelbrojt, Lucie Prod'homme.

${ }^{2}$ Conservatoire national de région, 10 rue Jean-Jacques Rousseau 74000 Annecy, jfavory@laposte.net

${ }^{3}$ http://www.labo-mim.org
} 
que ce qu'ils font ne fonctionne pas ou au contraire, comment montrer en quoi leur travail est satisfaisant. Ceci, sans intention de normalisation.

Comment aussi, donner à un auditeur les moyens lui permettant de « comprendre » l'évolution d'une pensée, qui après avoir privilégié les combinaisons de hauteurs et durées, s'est orientée vers l'utilisation des qualités morphologiques du son.

Des outils existent avec les «Objets Sonores » élaborés par Pierre Schaeffer et présentés dans son Traité des Objets Musicaux. Ils rendent possible l'utilisation d'un vocabulaire descriptif et d'une grille de classification des sons selon des critères morphologiques.

Une description qui demande une attitude d'écoute particulière, attitude que Schaeffer appelle «écoute réduite » puisqu'elle consiste à faire abstraction de toute signification causale ou associative et à écouter le son pour ses caractéristiques propres. L' «Objet Sonore» se détachant du flux audio acoustique par les lois de la forme (contraste, continuité, clôture). De la même façon que l'on peut décrire un objet qui se déploie dans l'espace selon sa forme, sa couleur, sa position, son encombrement spatial, son poids, son matériau grâce à la vue, l'écoute réduite permet de décrire les sons selon 7 critères morphologiques :

- la masse, le timbre harmonique, le grain (qui composent la Matière),

- la dynamique et l'allure (qui composent la Forme),

- le profil mélodique et le profil de masse (qui font référence à la notion de Variation),

- et de les classer selon 3 couples de critères :

- morphologique (on s'attache à la facture de l'objet d'une part, et à sa masse d'autre part),

- temporel (on considère la durée de l'objet d'une part et d'autre part les variations à l'intérieur de cette durée selon les critères précédents),

- structurel (on considère l'équilibre de l'objet, choisi parmi les structures possibles et, pour ce niveau structurel choisi, le degré plus ou moins grand d'originalitét).

Mais cette description typo-morphologique se trouve impropre à considérer la musique comme objet signifiant.

C'est en nous plongeant dans l'écoute du répertoire électroacoustique pour voir si des règles communes de conduite, règles implicites et non-normatives, pouvaient être dégagées que nous avons repéré des segments musicaux qui, tant au niveau morphologique que par ce qu'ils semblaient nous raconter, nous paraissaient proches. Ces segments nous les avons alors regroupés, grâce à cette proximité à la fois morphologique et de signification, dans des classes d'équivalences que nous avons appelés Unités Sémiotiques Temporelles (UST).

«Unités » car ce sont des fragments musicaux, « Sémiotiques » car ces fragments semblent porteurs d'un sens, souvent en relation d'homologie avec quelque chose d'extra-musical et «Temporelles » car le sens est fonction de la façon dont la matière sonore s'organise, évolue, dans le temps. Ce sens restant d'ailleurs perceptible même hors contexte musical.

\footnotetext{
${ }^{4}$ P. Schaeffer, Traité des objets musicaux, Paris, Seuil, 1966, p. 436.
} 
Si au départ nous avions abordé la question par les musiques électroacoustiques, nous nous sommes rapidement rendu compte que de tels segments musicaux se retrouvent tout autant dans des musiques instrumentales et vocales, sans distinction de genres, d'époques ni de lieux. Il faut préciser que lorsque nous travaillons sur des musiques notées, notre démarche reste fondée sur l'écoute, le rendu sonore, et non la partition et que l'interprétation est donc capitale.

Ainsi, pour un même passage musical interprété par plusieurs instrumentistes, il se peut que l'une des interprétations soit entendue comme une UST et non les autres. Cela est fonction de la mise en temps effective du fragment noté.

Un document papier comme celui que vous êtes en train de lire, n'est, par conséquent, certainement pas la meilleure forme de communication pour appréhender la réalité des UST.

Néanmoins, voici la fiche de description que nous avons élaborée pour l'UST « Contracté-Étendu » :

DESCRIPTION MORPHOLOGIQUE :

- Unité délimitée dans le temps à deux phases contrastées.

- Phase «Contracté » : matière de type discontinu et irrégulier.

- Phase «Étendu » : globalement uniforme.

DESCRIPTION SÉMANTIQUE :

D'abord sensation de compression, comme si on s'appuyait fortement contre un obstacle ; puis cet obstacle cède brutalement, supprimant la résistance et permettant à la force de se relâcher. Passage subit « d'énergie localisée » en « énergie diffuse ».

\section{AUTRES CARACTÉRISTIQUES PERTINENTES NÉCESSAIRES :}

- Phase «Contracté » : sa matière doit être plus rugueuse et plus épaisse que celle de la phase «Étendu », son profil dynamique est un crescendo rapide ou bien une intensité maximale immédiate ou une combinaison de sforzando.

- Phase «Étendu»: doit être entretenue volontairement et non être une simple résonance.

- L'entre-deux phases : ne peut être une trajectoire continue. Il y a une rupture.

- Les durées et les rapports des durées des deux phases ne sont pas indifférents : ils doivent permettre la perception du contraste.

Nous avons donc affaire à une configuration sonore constituée de deux phases : la première, brève et composée de petits événements accumulés, est enchaînée, après une très légère césure, à une seconde phase en contraste morphologique avec la première puisque lisse et étendue, distendue.

Au-delà de la description morphologique de cette séquence, ce qui la définit en propre c'est le sentiment qui s'en dégage de précipitation puis d'élongation du temps.

Nous avons ainsi isolé et défini 19 UST. Pour chacune d'elle il y a un intitulé comme «Contracté-Étendu» dans ce qui précède. Cet intitulé doit être considéré comme une étiquette et non comme la signification de l'UST. 
Ainsi, l'UST «Chute », telle que nous la considérons, fait-elle référence à la description que nous en donnons tant au niveau morphologique que sémantique. En même temps, si nous l'avons appelée «Chute», ce n'est pas anodin car il y une homologie avec la sensation vécue de la chute physique. Bien que nous ayons aussi trouvé des «Chutes » musicales qui s'affranchissent des lois de la gravitation, puisque pouvant monter dans la tessiture. Aller du grave vers l'aigu. Ce qui compte, c'est que ce mouvement se fasse en accélérant. Une forme d'aspiration en quelque sorte.

Il en va ainsi pour toutes les UST avec leur intitulé.

Voici notre définition de la « Chute » :

\section{DESCRIPTION MORPHOLOGIQUE :}

- Unité délimitée dans le temps à deux phases.

- $1^{\mathrm{e}}$ phase : globalement uniforme, même si la matière est animée d'un mouvement interne.

- $2^{\mathrm{e}}$ phase : comporte un mouvement d'accélération et évolue en hauteur soit en montant, soit en descendant.

\section{DESCRIPTION SÉMANTIQUE :}

- Équilibre instable qui se rompt.

- Suspens puis basculement (la prise de conscience de la phase de suspens se fait, en fait, après coup). Perte d'énergie potentielle qui se convertit en énergie cinétique.

\section{AUTRES CARACTÉRISTIQUES PERTINENTES NÉCESSAIRES :}

- La $2^{\mathrm{e}}$ phase ne peut pas évoluer de façon uniforme. Elle doit comporter une accélération mais pas nécessairement de variation de hauteur.

- Le passage de la $1^{\mathrm{e}}$ à la $2^{\mathrm{e}}$ phase se fait par changement brusque («point anguleux ») et non de façon continue.

- Une continuité (et pas forcément une identité) de matière est nécessaire entre les deux phases.

- «Ça » bascule. Il y a un « ça » reconnaissable, commun aux deux phases.

La durée globale ne doit pas excéder quelques secondes de façon à être intégrée perceptivement comme une forme.

Si vous avez en tête le tableau de Bruegel intitulé « La paraboles des aveugles », il constitue une bonne représentation de notre définition de la "Chute », la dimension dynamique étant suggérée dans la représentation picturale alors qu'elle est réalisée dans les UST sonores.

Il y a deux grandes catégories d'UST : celles à durée déterminée (comme le «Contracté-Étendu » et la «Chute ») qui sont stockées dans la mémoire immédiate, et celles à durée indéterminée, qui pourraient durer indéfiniment mais dont l'écoute d'un simple extrait permet d'en saisir le sens. Si les premières sont souvent en relation avec des sensations de mouvements, de forces dynamiques, d'énergie qui évolue au cours du temps, les secondes font plus appel à des descriptions d'états. 
- Ainsi «En suspension » :

DESCRIPTION MORPHOLOGIQUE :

Unité non délimitée dans le temps, constituée d'une formule en répétition quasiment sans variation, dans un déroulement temporel assez lent, et dont la matière sonore et/ou les événements évoluent peu.

DESCRIPTION SÉMANTIQUE :

- Sans direction et en flottement.

- Équilibre des forces en présence donnant une sensation d'immobilité liée à un sentiment d'attente hésitante.

- On sait qu'il va arriver quelque chose, on ne sait ni quand ni quoi.

AUTRES CARACTÉRISTIQUES PERTINENTES NÉCESSAIRES :

Irrégularité ou absence des points d'appui temporels.

- Ou encore « Obsessionnel » :

DESCRIPTION MORPHOLOGIQUE :

Unité non délimitée dans le temps, à phase unique, constituée d'une formule dont la répétition (éventuellement variée) crée une pulsation.

\section{DESCRIPTION SÉMANTIQUE :}

- Caractère insistant.

- Procédé mécanique de répétition qui s'impose et sur lequel il semble qu'on ne puisse intervenir.

\section{AUTRES CARACTÉRISTIQUES PERTINENTES NÉCESSAIRES :}

- Importance d'un profil dynamique typé de la formule de base.

- La durée de la formule doit être suffisamment courte pour faire référence à une pulsation.

Il est intéressant de remarquer que ces deux dernières UST sont très proches sur le plan morphologique mais diffèrent au niveau sémantique. «Obsessionnel » est porteur d'un aspect mécanique sur lequel on ne peut intervenir alors que «En suspension » fait appel à un sentiment d'attente lié à une tension. Néanmoins, dans les deux cas il en ressort une sensation d'un temps immobile.

Le premier champ d'application des UST est l'analyse musicale. Des mises à l'épreuve de l'outil face à la complexité de la réalité musicale ont été réalisées ${ }^{5}$ avec, pensons-nous, succès. Bien que nous ne prétendions pas qu'il puisse s'adapter à toutes les musiques. Mais des incursions dans le champ de la création et de l'interprétation semblent tout autant valider l'outil qui trouve aussi des applications ailleurs qu'en musique, comme en danse ou dans l'image animée. En fait, toutes choses où l'écoulement du temps est structurant.

\footnotetext{
${ }^{5}$ Les Unités Sémiotiques Temporelles-Nouvelles clés pour l'écoute (cédérom MIM).
} 